\title{
Effectiveness of multifaceted educational programme to reduce antibiotic dispensing in primary care: practice based randomised controlled trial
}

\author{
(c) (1) (8) OPEN ACCESS
}

\section{Christopher C Butler professor of primary care medicine ${ }^{1}$, Sharon A Simpson senior research} fellow $^{12}$, Frank Dunstan professor of medical statistics ${ }^{1}$, Stephen Rollnick professor of healthcare communication $^{1}$, David Cohen professor of health economics ${ }^{3}$, David Gillespie statistician ${ }^{12}$, Meirion $\mathrm{R}$ Evans ${ }^{1}$, senior lecturer in epidemiology and public health, M Fasihul Alam research fellow ${ }^{3}$, Marie-Jet Bekkers research associate ${ }^{12}$, John Evans information manager ${ }^{12}$, Laurence Moore professor of public health improvement ${ }^{4}$, Robin Howe consultant microbiologist ${ }^{5}$, Jamie Hayes director, Welsh medicines resource centre ${ }^{6}$, Monika Hare trial manager ${ }^{12}$, Kerenza Hood professor and director ${ }^{12}$

${ }^{1}$ Institute of Primary Care and Public Health, School of Medicine, Cardiff University, Cardiff, CF14 4XN, UK; ${ }^{2}$ South East Wales Trials Unit, School of Medicine, Cardiff University, Cardiff; ${ }^{3} \mathrm{Health}$ Economics and Policy Research Unit, University of Glamorgan, Pontypridd; ${ }^{4} \mathrm{Cardiff}$ Institute of Society and Health, School of Social Sciences, Cardiff University, Cardiff; ${ }^{5}$ National Public Health Service Microbiology Cardiff (Velindre NHS Trust), University Hospital of Wales, Cardiff; ${ }^{6}$ Welsh Medicines Resource Centre (WeMeReC), Academic Centre, University Hospital Llandough, Penarth

\begin{abstract}
Objective To evaluate the effectiveness and costs of a multifaceted flexible educational programme aimed at reducing antibiotic dispensing at the practice level in primary care.

Design Randomised controlled trial with general practices as the unit of randomisation and analysis. Clinicians and researchers were blinded to group allocation until after randomisation.

Setting 68 general practices with about 480000 patients in Wales, United Kingdom.

Participants 34 practices were randomised to receive the educational programme and 34 practices to be controls. 139 clinicians from the intervention practices and 124 from control practices had agreed to participate before randomisation. Practice level data covering all the clinicians in the 68 practices were analysed.

Interventions Intervention practices followed the Stemming the Tide of Antibiotic Resistance (STAR) educational programme, which included a practice based seminar reflecting on the practices' own dispensing and resistance data, online educational elements, and practising consulting skills in routine care. Control practices provided usual care.

Main outcome measures Total numbers of oral antibiotic items dispensed for all causes per 1000 practice patients in the year after the intervention, adjusted for the previous year's dispensing. Secondary
\end{abstract}

outcomes included reconsultations, admissions to hospital for selected causes, and costs.

Results The rate of oral antibiotic dispensing (items per 1000 registered patients) decreased by 14.1 in the intervention group but increased by 12.1 in the control group, a net difference of 26.1. After adjustment for baseline dispensing rate, this amounted to a $4.2 \%$ (95\% confidence interval $0.6 \%$ to $7.7 \%$ ) reduction in total oral antibiotic dispensing for the year in the intervention group relative to the control group $(P=0.02)$. Reductions were found for all classes of antibiotics other than penicillinase-resistant penicillins but were largest and significant individually for phenoxymethylpenicillins (penicillin V) $(7.3 \%, 0.4 \%$ to $13.7 \%)$ and macrolides (7.7\%, $1.1 \%$ to $13.8 \%)$. There were no significant differences between intervention and control practices in the number of admissions to hospital or in reconsultations for a respiratory tract infection within seven days of an index consultation. The mean cost of the programme was £2923 (€3491, \$4572) per practice (SD £1187). There was a $5.5 \%$ reduction in the cost of dispensed antibiotics in the intervention group compared with the control group (-0.4\% to $11.4 \%)$, equivalent to a reduction of about $£ 830$ a year for an average intervention practice.

Conclusion The STAR educational programme led to reductions in all cause oral antibiotic dispensing over the subsequent year with no significant change in admissions to hospital, reconsultations, or costs. Trial registration ISRCT No 63355948. 


\section{Introduction}

Antibiotic resistance is one of the most important public health issues of our time, and antibiotic use is an important factor in the increase in resistance at the population and individual level. ${ }^{12}$ As most antibiotics are prescribed in primary care and many of these prescriptions for conditions such as bronchitis, otitis media, purulent rhinitis, and sinusitis are of questionable value, reducing inappropriate prescribing in this setting is an important goal. ${ }^{3-7}$ Antibiotic use increases an individual's risk of carrying and being infected by resistant organisms rather than sensitive organisms, with the greatest effect occurring immediately after treatment but persisting for up to 12 months. ${ }^{28}$ In a systematic review and meta-analysis of five studies of urinary tract bacteria that included 14348 participants, the pooled odds ratio for resistance was 2.5 (95\% confidence interval 2.1 to 2.9 ) within two months of antibiotic treatment and 1.3 (1.2 to 1.5$)$ within 12 months. In seven studies of respiratory tract bacteria that included 2605 participants, pooled odds ratios were 2.4 (1.4 to $3.9)$ and 2.4 (1.3 to 4.5$)$ for the same periods. ${ }^{2}$ In addition to driving resistance, inappropriate prescribing of antibiotics wastes resources, unnecessarily exposes people to the risk of side effects, and encourages future consulting. ${ }^{9}{ }^{10}$ Antibiotic resistance is common in primary care, causes symptoms to last for longer, ${ }^{11}$ and is associated with higher drug and reconsultation costs. ${ }^{12}$ Conserving antibiotic sensitivity through more appropriate use is a priority. ${ }^{13}$ Importantly, reducing antibiotic prescribing has been associated with a reduction in antimicrobial resistance. ${ }^{14-16}$

Although antibiotic prescribing to ambulatory patients decreased by about a third in most developed countries during the 1990s, the use of broad spectrum agents has increased in the United States, ${ }^{17}$ and antibiotic prescribing has increased since 2000 in primary care in the United Kingdom. ${ }^{18}{ }^{19}$ For example, there was a $10 \%$ increase in antibiotic prescribing for children between 2003 and 2006. ${ }^{1819}$

Recent public campaigns on antibiotic awareness in the UK have not been associated with reductions in all cause antibiotic prescribing in primary care. ${ }^{20}$ There is wide variation between general practices in antibiotic prescribing rates that cannot be explained by differences in the epidemiology of infections, populations, or case mix. ${ }^{121}{ }^{22}$ Further gains could therefore safely be made by reducing inappropriate antibiotic prescribing. ${ }^{9}$ Interventions are likely to be most effective if they are multifaceted and broadly applicable across a range of infections in all age groups, rather than focused on single strategies for single conditions in single age groups (such as a guideline for managing sore throat in children). ${ }^{23}$

Changing clinicians' prescribing behaviour, however, is a complex matter. ${ }^{24}$ Guidelines have limited effect, even when clinicians have been involved in their development. ${ }^{25} 26$

Systematic reviews ${ }^{24} 27-29$ of interventions to change clinician behaviour have found that successful interventions are most likely to be multifaceted, based on an assessment of barriers to change, responsive to local circumstances, and focused, and to incorporate an active educational outreach component including skills development and to resonate with clinicians' values. ${ }^{30} 31$ Systematic reviews of interventions to enhance the quality of antibiotic prescribing practices in ambulatory care have also found that multifaceted interventions are likely to be most effective..$^{23}{ }^{32}$ Blended learning interventions offer variety, flexibility, and convenience and are increasingly promoted, but their effectiveness in enhancing antibiotic prescribing has not been assessed. Interventions that are informed by theories of behaviour change are likely to be more effective. ${ }^{33}$ For example, shared decision making with patients is being promoted and achieves more conservative antibiotic prescribing in efficacy studies. ${ }^{34-37}$ Reducing antibiotic prescribing in primary care, however, should not be at the expense of increased reconsultations, admission to hospital, or, ideally, costs.

We developed the Stemming the Tide of Antibiotic Resistance (STAR) educational programme to be multifaceted (e-learning, practice based outreach, experiential learning and reflections), responsive to local circumstances (feedback of practice level data on antibiotic dispensing and resistance), and based on previously identified recommendations (practice-wide effort; time efficient consultation strategies that "enable" patients and maintain relationships). ${ }^{38-40}$ It used a phased engagement and implementation process to achieve certification on completion of a learning portfolio and then sought to maintain change through a web based exchange of experiences. ${ }^{40}$ Based on social learning theory, the intervention attempted to develop clinicians' sense of importance about change (the "why" of change) and their confidence in their ability to achieve change (the "how" of change). ${ }^{41}{ }^{42}$ We determined whether the STAR programme could reduce antibiotic dispensing for all causes at the practice level in primary care without increasing reconsultations, admissions to hospital for selected causes, and costs.

\section{Methods}

\section{Setting}

The study was conducted in general medical practices in Wales, UK, in 2007 and 2008 (see below). In the UK people, regardless of age, are registered with a general practice and consult with that practice for their primary care unless there are unusual circumstances such as being away from home or direct attendance at an emergency department, usually in the event of an acute more serious illness. Registered patients constitute the practices' lists and these vary over time for each practice. Part of general practitioners' payments is determined by list sizes so these data are generally kept up to date.

\section{Study design}

We randomised general practices to intervention or control (usual care). As patients registered at a practice can be managed by any of the clinicians in the practice, we considered the practice as the unit for randomisation and analysis. The main outcome measure was the rate of dispensing oral antibiotics for any cause over one year for the whole practice population. Clinicians provided consent to participate, but consent from individual patients was not required. The details of the trial design have already been published. ${ }^{43}$

\section{Randomisation and blinding}

Randomisation took place once all practices were recruited and all participating clinicians had provided written consent. This allowed us to use dynamic block allocation ${ }^{44}{ }^{45}$ to achieve balance between groups of practices for the potential confounders of previous rate of antibiotic dispensing (averaged over the past year), practice size (number of whole time equivalent staff at recruitment), and proportion of clinicians in the practice registered for the study. The practices were divided into three sets of 24, 22, and 22 practices; within each set we generated all possible allocations into two groups and selected the 1000 allocations within each set with the best balance with respect to the specified confounders. The independent statistician on the trial steering committee selected one allocation at random for each set and randomly assigned intervention or control to 
the two groups in each set to construct the final allocation. Thus, all were blinded to group allocation until after randomisation.

\section{Intervention}

This intervention was a blended learning experience for participants as it was a combination of various learning methods and topics (reflection on own practice, provision of new research evidence and guidelines, video-rich material presenting novel communication skills based on motivational interviewing, practice in usual clinical contexts, sharing experiences and views on a web form, and participating in a facilitator led, practice based seminar). The box contains a full description, and figure $1 \Downarrow$ describes the online structure of the programme. Novel aspects include clinicians reflecting on their practice's own data on antibiotic dispensing and resistance, novel communication skills derived from principles of motivational interviewing, and sharing reflections on actual practice with these skills. The intervention was flexible in that clinicians could access the online components and try out new skills with their patients at times that were convenient to themselves.

The control practices were not exposed to the learning programme and provided care as usual.

\section{Outcomes}

We used data from the Prescribing Audit Reports and Prescribing Catalogues (PARC) (www.nhsbsa.nhs.uk/ prescriptions) to determine the primary outcome for each practice: the total number of dispensed oral antibiotic items per 1000 registered patients for the year after the intervention practices were exposed to the STAR programme. The database contains data for dose and the number/volume of antibiotics but not the defined daily dose or clinical data. The version of this database in England has been successfully used for assessing effects of large scale interventions for reductions in antibiotic prescribing. ${ }^{50}$ If two antibiotics were prescribed for the same person at the same consultation, this would be registered as two items.

Our secondary outcomes were hospital admission rates, practice reconsultation rates, and costs. We examined hospital admission rates for possible respiratory tract infections and their complications per 1000 registered patients for the year after the intervention practices were exposed to the programme. We chose these infections because they are the most common indication for prescribing unnecessary antibiotics in primary care. We used routinely collected data from the Patient Episode Database for Wales (PEDW), which records inpatient/day case care for Welsh residents treated in an NHS hospital anywhere in the UK (www.hsw.wales.nhs.uk/page.cfm?orgid=166\& pid=4262).

A single admission to hospital for a complication was deemed to have occurred if a patient had been admitted to hospital for a possible respiratory tract infection or complication. If a patient was admitted more than once, and the gap between admissions was 30 days or more, this was considered to be a separate complication episode.

In patients with respiratory tract infections, we identified practice reconsultation rates using diagnostic READ codes (www.connectingforhealth.nhs.uk/systemsandservices/data/ uktc/readcodes) recorded by the general practitioner over seven days, 14 days, and 31 days after an initial consultation. These data were collected from the electronic records of a subsample of 37 general practices (20 intervention and 17 control). We selected practices for this subsample on the basis of the computer software systems they used for their clinical records based on ease of extraction and resource required (Vision (www.nvug. org/index.html), Ganymede (www.isofthealth.com/en-GB/ Solutions/UK\%20Primary\%20Care/Ganymede.aspx).

To assess costs, we prospectively recorded all resources used in preparation for STAR training in relevant units, multiplied by appropriate unit costs, ${ }^{51}$ and apportioned equally across intervention practices. Seminars were assumed to last for 1.5 hours and were delivered at a flat fee per seminar of $£ 500$ ( $€ 597$, \$780) plus travel costs.

Learning times at seminars and on line were valued at relevant unit costs. It was assumed that all log-on time was during working hours, as would be the case if STAR were rolled out across healthcare systems. Prices of antibiotics dispensed were from the British National Formulary. ${ }^{52}$ Costing was from a health service perspective and all costs are in 2009 prices. As the follow-up period for costs and effects apart from resistance was one year, discounting was not applied.

Data on antibiotic dispensing, hospital admissions, and reconsultations were collected through routine administrative systems that were not influenced by the STAR study research process.

\section{Statistical analysis Sample size calculation}

The standard deviation between practices of the changes within practices over successive years in total dispensed antibiotic rates per 1000 patients was 70 items per 1000 patients in Wales. To detect a possible difference of $10 \%$ (73 dispensed oral antibiotic items per 1000 patients) in the change in total oral antibiotic dispensing rate per year between intervention and control practices, with $90 \%$ power, we required 21 practices per group. Increasing this to over 30 practices per group, however, allowed the study to detect a difference of $8 \%$ in antibiotic dispensing with $90 \%$ power and gave more power for analysis of secondary outcomes.

\section{Main analyses}

The main analysis was intention to treat and compared the two groups' annual rates of total oral antibiotic dispensing for all causes per 1000 practice patients within practices in the year after the intervention, by using analysis of covariance with the previous year's prescribing as a covariate. Because the distribution of rates was positively skewed, these were log transformed to produce approximately normally distributed data, enabling us to calculate confidence intervals for the ratios of dispensing rates between the two groups rather than the differences. The assumption of constant variance was not satisfied as the list sizes varied across practices from less than 2000 to nearly 17000 and the precision of the rate varied accordingly. To account for this, we weighted the data using the log of the list sizes. The same methods were applied to antibiotic costs.

The follow-up period started for each practice in the intervention group from the month after their practice based seminar (between May and October 2007) and continued for 12 months. We chose a control practice at random for each practice in the intervention group and assigned the same year long baseline and follow-up period.

We similarly compared secondary outcomes of average hospital admission rates for specified complications between the two groups for the whole year. Reconsultation rates were compared with Mann-Whitney U tests as the data were not normally distributed. We calculated the median difference in 


\section{Detailed description of the study intervention}

The structure and online content of the STAR educational programme can be viewed at www.stemmingthetide.org. The entire learning programme consists of seven parts.

- Part 1 (on line): Clinicians were asked to make judgments about how they might handle four case scenarios (fig $2 \downarrow$ ). They also reflected on the problem of antibiotic resistance and their decisions regarding antibiotic prescribing and were provided with up to date summaries of research evidence and guidelines as well as videos giving a range of opinions (viewpoints from experts, patients, and clinicians)

- Part 2 (on line): Clinicians reflected on their decision whether or not to prescribe antibiotics for four actual patients they had managed and uploaded summaries of the cases onto the STAR website. The researchers did not limit the type of infections. Other clinicians taking part in the study could also see these summaries

- Part 3 (face to face): Guided by the principles of information exchange derived from motivational interviewing, ${ }^{46}$ a facilitator in a practice based seminar presented, and invited interpretation of, seven year trends for antibiotic dispensing and resistance trends at all Wales local area level, and the actual practice (fig $3 \Downarrow$ ). Resistance rates were based on coliforms in urine samples routinely submitted to laboratories from general practice for microbiological analysis (resistance in urinary tract infection is associated with prescribing for other infections and large numbers of urine samples are routinely sent for microbiological analysis). ${ }^{14}$ The aim was to encourage prescribers to interpret data from their own practice and to consider appropriate responses

- Part 4 (on line): Clinicians repeated questions on the four initial online case scenarios and attitude questions from part 1 . They were able to access responses of other participating clinicians and compare them with their own. Attention then turned to consulting skills. ${ }^{354}$ These included: "Lifting the Lid"-identifying the main concerns and expectations of the patient; "Information Exchange"-using a strategy from motivational interviewing to share information about the pros and cons of antibiotic use, prognosis, treatment, and reconsulting; and "Wrap-Up"-acknowledging the patient's concerns, summarising the medical situation, clarifying reasons to reconsult, and checking back with the patient. ${ }^{47} 48$ Four video scenarios demonstrated these skills, and interactive exercises invited clinicians to identify evidence of "good practice in an antibiotic consultation" by clicking on option boxes as videos unfolded. Perspectives from patients, clinicians, and expert colleagues on the consultations were linked to supporting research evidence and guidelines

- Part 5 (clinical practice with reflection): In keeping with principles of context bound learning ${ }^{49}$ clinicians described three of their own consultations in which they used the new consultation skills. These reflections were recorded on line and securely shared with other clinicians on the programme

- Part 6 (online): An ongoing active web forum provided updates on emerging relevant scientific evidence and allowed educators in the STAR study team to respond to queries, feedback, and comments. Clinicians were able to add to existing threads and start new topics on the forum

- Part 7 (on line): A booster module, six to eight months after completion of the initial training, reminded clinicians of the previously outlined consultation skills and showed a video of a consultation for a common infection where they were asked to identify key strategies that were used. Clinicians were sent a snapshot of their practice's antibiotic dispensing from two recent winter months compared with the rates of corresponding months for the year before they began the programme.

reconsultation rates between intervention and control groups with $95 \%$ confidence intervals constructed with bootstrapping methods.

\section{Results}

We wrote to 212 of 499 general practices in Wales about the study. Forty five practices were ineligible because we could not obtain certain routine data for them. We had just contacted 242 practices, chosen at random from the 499 , about a different study and so also considered them ineligible for this study. Of the 212 practices we wrote to, 102 expressed an interest in taking part. After discussion, 70 agreed to participate. One practice subsequently withdrew before randomisation, and one was considered ineligible because only one out of seven clinicians agreed to participate, leaving 68 practices serving about 480 000 patients that were randomised to either intervention or control (34 each) (fig $4 \Downarrow$ ). The previous year's antibiotic dispensing rate for the 68 practices that were randomised was about $15 \%$ lower than the Welsh average.

Two practices, one in each group, withdrew after randomisation but were included in the intention to treat analyses. In the intervention practices that agreed to participate, 127 of the 139 clinicians completed the programme. Of the 124 clinicians in the control practices, 117 participated (fig 4 ). Table $1 \Downarrow$ describes the demographic features of the practices in each randomisation group.

\section{Uptake of the intervention}

Intervention clinicians had to complete each online learning component before the software would allow them to access the next. All but one completed the four parts of the online training and uploaded descriptions of consultations for the portfolio tasks. Only 10 clinicians did not attend the practice based seminars. Seventy six completed the optional booster session at six months. Eleven clinicians entered new threads on the online forum but there were 81 posts and 1485 viewings of these posts and threads.

\section{Antibiotic dispensing, admissions to hospital, reconsultation, and costs \\ Antibiotic dispensing}

General practices exposed to the programme reduced oral antibiotic dispensing for their patients by 14.1 items per 1000 registered patients, while control practices increased antibiotic dispensing by 12.1 items per 1000 registered patients. Thus, the intervention led to a difference in 26.1 items per 1000 registered patients per year. From the weighted analysis of covariance model, we found a significant reduction in antibiotic dispensing in the intervention group, after controlling for the baseline dispensing rate $(\mathrm{P}=0.02)$. This represented a reduction of $4.2 \%$ (95\% confidence interval $0.6 \%$ to $7.7 \%$ ) in total oral antibiotic dispensing for all age groups and conditions in the year after intervention (table $2 \Downarrow$ ) in the intervention group relative to the control group. Reductions were found for all classes of antibiotic other than penicillinase-resistant penicillins but were largest and significant individually for phenoxymethylpenicillins (7.3\%, $0.4 \%$ to $13.7 \%$ ) and macrolides $(7.7 \%, 1.1 \%$ to $13.8 \%$ ) (table 2).

We had hypothesised that the effect would be greater in those intervention practices where two thirds or more of the clinicians participated. The average reduction in dispensing in the follow-up year in those practices in the intervention group in which more than $67 \%$ of general practitioners participated was 17.7 items per 1000 registered patients per year, while in the remainder of the intervention group dispensing actually increased by an average of 2.6 items per 1000 registered patients per year.

\section{Admissions to hospital and reconsultations}

There were no significant differences in admissions between intervention and control groups. The rate of hospital episodes 
for possible respiratory tract infections and complications in the intervention group increased by $1.9 \%$ ( $-8.2 \%$ to $13.2 \%)$ relative to the control group, in the year after the intervention $(\mathrm{P}=0.72$; table $2 \Downarrow)$.

There were no significant differences between intervention and control groups for reconsultation rates after an index consultation, expressed as reconsultations per 1000 registered patients, for respiratory tract infections (median difference (intervention-control) -0.65 ( -1.69 to 0.55$)$ at seven days; -1.33 ( -2.12 to 0.74$)$ at 14 days; and $-2.32(-4.76$ to 1.95$)$ at 31 days (table $3 \Downarrow$ ).

\section{Costs}

Table $4 \Downarrow$ shows the costs of delivering STAR training to 33 intervention practices. The average time clinicians spent on line was 238 minutes. The opportunity cost of those trained (value of time not spent on other work activities) represents the largest cost element of the programme (£71 659). The overall mean cost was $£ 2923$ per practice (SD £1187).

The mean annual cost of antibiotic dispensing fell in both groups between baseline and follow-up (table 2), but the fall in intervention practices was greater (intervention $£ 120.76$, control $£ 2.31$ per 1000 patients). The mean antibiotic cost for the follow-up year in the intervention group decreased by $5.5 \%$ $(-0.4 \%$ to $11.4 \% ; \mathrm{P}=0.07)$ relative to that in the control group. Thus, the best estimate of the difference in antibiotic dispensing costs is $£ 118.45$ per 1000 patients per year or about $£ 830$ per year less for an average intervention practice.

\section{Discussion}

General practices exposed to the STAR educational programme reduced their rate of dispensing oral antibiotics for all causes by 14 items per 1000 registered patients, while control practices increased theirs by 12 items per 1000 registered patients. Thus, the intervention led to a difference of 26 items per 1000 registered patients a year. This represented a reduction of $4.2 \%$ $(0.6 \%$ to $7.7 \%)$ in total oral antibiotic dispensing for all age groups and all conditions in the year after the intervention relative to the control practices. Reductions were significant for antibiotics commonly used for certain respiratory tract infections (such as sore throat) in the UK $(7.3 \%$ for phenoxymethylpenicillins (penicillin $\mathrm{V}$ ) and $7.7 \%$ for macrolides). The intervention incorporated use of the modified Centor criteria, ${ }^{53}$ which could explain the greater impact on antibiotics commonly used for treating sore throats. The reduction was greater in the practices where more than two thirds of the general practitioners participated in the study. The increased dispensing in the control practices could reflect increasing antibiotic prescribing across the UK. ${ }^{18} 19$

We found no evidence that antibiotic dispensing was reduced at the expense of increased reconsultations or complications with common infections, although the study was not powered to identify small changes.

The reductions in antibiotic dispensing were achieved at an average cost of $£ 2923$ per practice (SD $£ 1187$ ). There was a $5.5 \%$ non-significant reduction in the cost of dispensed antibiotics in the intervention group, amounting to $£ 118.45$ per 1000 patients per year. If this was the true value and the benefit of the intervention was maintained over time, then the investment in the intervention would be recouped within 3.5 years.

\section{Strengths and limitations of the study}

We showed that a complex flexible blended learning approach to continuing education for clinicians is effective in reducing all cause antibiotic prescribing, and we evaluated the impact on the costs of antibiotic dispensing. ${ }^{23} 32$

Some primary care clinicians consider antibiotic resistance to be a hospital based problem, unaffected by their own prescribing behaviour. ${ }^{38}{ }^{39}$ The intervention included discussion of practice specific antibiotic dispensing and resistance data, as well as a report showing an association between reductions in practice based dispensing and local levels of resistance, which has not been done before. ${ }^{14}$

Increased use of "delayed prescribing" in the UK makes dispensed antibiotics a better outcome measure for this study than prescribed antibiotics as it is a better proxy for antibiotics actually consumed.

The outcome was antibiotic dispensing for the whole practice population regardless of how many practitioners in the practice were exposed to the intervention. Our pragmatic approach meant that we used practices in which not all clinicians participated. This enhances the applicability of findings as not all clinicians are likely to participate should the intervention be rolled out. Studies that analyse data only from participating clinicians are likely to overestimate potential practice effects.

Selection of patients and consent from individual patients was not required, and outcomes were collected for the whole practice population irrespective of diagnosis by using routinely collected data. This eliminated the problem of limiting the primary outcome to only those patients who participating clinicians were able to recruit, rather than determining the practice level effect. ${ }^{54}$ Furthermore, diagnostic codes can be selected to justify decisions regarding antibiotic prescribing (diagnostic drift), which can make judgments of appropriate prescribing difficult. It is potentially much easier to show changes for small numbers of individually recruited patients over a short period of time than for all cause prescribing for a whole practice population over a whole year. ${ }^{36}{ }^{37} 55$ Other studies have included only three winter months in their outcomes. ${ }^{50}$ This could explain why reductions seen in our study are less dramatic than those seen in efficacy studies.

Few, if any, studies on this issue have evaluated effects of interventions on complications and repeat consultations. ${ }^{23} 32$ Our limited analyses did not identify a significant effect on admission to hospital for respiratory tract infections or complications. These are relatively rare, however, and the study was not powered to detect small differences. We also examined effects on reconsultations for selected common infections in a subset of practices and found no important differences; in fact the observed rate was lower in the intervention group.

We conducted process evaluation interviews with 31 purposively sampled clinicians in the intervention practices. ${ }^{56}$ Most reported increased awareness of antibiotic resistance, greater self confidence in reducing antibiotic prescribing, and at least some change in consultation style and antibiotic prescribing behaviour. Reported practice changes included adopting a practice-wide policy of reducing antibiotic prescription. Many reported increased insight into patients' expectations, ultimately contributing to improved clinician-patient rapport. The components of the intervention reported as having the greatest influence on changing clinician behaviour were the up to date resources for evidence from research, simple and effective communication skills presented in online videos, and presentation of the practice's own antibiotic dispensing rates 
combined with practice specific and local area data on resistance. ${ }^{56}$

As we do not have reliable patient denominator data for each practitioner (numbers of patients registered or seen, and the diagnosis), we could not determine which practitioners responded most to the intervention and for which patients and diagnoses the greatest reductions were achieved. The lack of diagnostic data means that we were able to consider only reductions in total oral antibiotics and not the effect on inappropriate antibiotic prescribing. Also, we do not know if hospital admissions were increased among those who might otherwise have been prescribed antibiotics in primary care. We were unable to provide a meaningful comparison of the length of consultations before and after the intervention.

While almost all intervention clinicians completed the different components of the intervention, only 11 entered new threads on the web forum (part 6). There were, however, 81posts (comments on new threads and responses to comments) and over 1400 viewings of the threads and subsequent posts.

\section{Comparison with other studies}

National public awareness campaigns in the UK around the time this study was done were not associated with any reduction in all cause antibiotic prescribing in the community or changes in the public's reported behaviour and beliefs..$^{20} 57$ A regional mass media campaign in northeast England over two years included leaflets, posters on buses, local newspaper, radio, and TV coverage, and professional education and prescribing support. ${ }^{50}$ They found 21.7 fewer items were prescribed per 1000 population for the intervention population over the winter months compared with our finding of 26 fewer items for a whole year.

A systematic review of interventions to reduce unnecessary antibiotic prescribing included 43 studies and found that most (38) focused only on acute respiratory infections. ${ }^{23}$ Most of these studies evaluated the effect of only one or two types of approach, in contrast with our intervention, which explored both the "how" and "why" of change in a flexible learning format, using practice-specific prescribing and resistance data, information about guidelines, reflections on practice based experience, academic outreach, and a web forum. None of the other studies provided feedback to practices about their own antibiotic dispensing rates combined with practice specific resistance data. Our intervention combines all of the elements identified as promising or effective and provides cost data. ${ }^{23}{ }^{32} \mathrm{Few}$ of the studies included in systematic reviews provided longer term follow-up data. Effect sizes vary considerably in the studies included in the reviews, which is expected given the heterogeneous study designs, interventions, and outcome measures..$^{23}{ }^{32}$ This also makes meaningful comparisons about the relative effectiveness of studies difficult.

\section{Conclusions}

The STAR educational programme led to reductions in oral antibiotic dispensing for all causes over the subsequent year with no significant changes in admissions to hospital, reconsultations, or costs. These results will be of interest to those concerned with antimicrobial stewardship and in the broader project of enhancing the quality of prescribing and changing clinicians' behaviour through innovation in continuing medical education. of Cardiff University, who was the study administrator, and Patrick Cannon, Valerie Dougall, James Goldsmith, and Dan Bruce of the Healthcare Learning Company, London, who designed and produced the online learning environment. We thank the independent members of the trial steering committee, Ian Williamson (chair), Alistair Hay, and Alan Montgomery (independent statistician).

Contributors: CCB conceived the study, acted as principal investigator, and led the protocol development, study implementation, interpretation, and report writing. SAS contributed to protocol development and study implementation, including a special contribution to the intervention development, interpretation, and report writing. FD contributed to the design of the study, data management, interpretation of the results, and writing, revised the report, and led aspects of the statistical analysis. SR contributed to protocol development and report writing and conceived the learning programme structure and directed its development. DC contributed to protocol development, interpretation, and report writing and planned and led the analyses of costs. MRE contributed to protocol development, study implementation, and data interpretation and assisted in drafting the manuscript. MFA contributed to the economic statistical analysis and interpretation of data and assisted in drafting the manuscript. M-JB was the trial manager for part of the study, led on the process evaluation interviews, and contributed to report writing. JE acted as the data manager, liaised with providers regarding data collection, developed and implemented extraction methods from primary care systems, contributed to the data management, study implementation, and report writing. DG contributed to the data management, statistical analysis and interpretation of the data, report writing, and drafting of the manuscript. LM contributed to protocol development and reviewed the manuscript. $\mathrm{RH}$ contributed to the study design, with a particular focus on outcome measurement. He helped develop the seminar intervention and contributed to the report writing. $\mathrm{JH}$ contributed to the development and led the delivery of the face to face component of the intervention, interpretation and draft manuscripts. MH contributed to study implementation, data collection, and interpretation and report writing, and was the trial manager for part of the study. $\mathrm{KH}$ contributed to the design of the study, data management, statistical analysis and interpretation of the results, and writing the report. All authors have given final approval of the submitted manuscript. CCB is guarantor.

Funding: This study was funded by the UK Medical Research Council (G0500956). The South East Wales Trials Unit is funded by the National Institute for Health and Social care Research, which also provide service support costs.

Competing interests: All authors have completed the ICMJE uniform disclosure form at www.icmje.org/coi_disclosure.pdf (available on request from the corresponding author) and declare: no support from any organisation for the submitted work; no financial relationships with any organisations that might have an interest in the submitted work in the previous three years; no other relationships or activities that could appear to have influenced the submitted work.

Ethical approval: The study was approved by the multicentre research ethics committee (MREC 06/MRE09/31) and all local health boards in Wales. Consent was obtained from clinicians but not from individual patients, but the presented data are anonymised and risk of identification is low.

Data sharing: No additional data available.

1 Goossens H, Ferech M, Vander Stichele R, Elseviers M. Outpatient antibiotic use in Europe and association with resistance: a cross-national database study. Lancet 2005;365:579-87.

2 Costelloe C, Metcalfe C, Lovering A, Mant D, Hay AD. Effect of antibiotic prescribing in primary care on antimicrobial resistance in individual patients: systematic review and meta-analysis. BMJ 2010;340:c2096.

3 Glasziou P, del Mar C, Sanders S, Hayem M. Antibiotics for acute otitis media in children Cochrane Database Syst Rev 2004;1:CD000219.

4 Del Mar CB, Glasziou PP, Spinks AB. Antibiotics for sore throat. Cochrane Database Syst Rev 2006;4:CD000023.

5 Arroll B, Kenealy $\mathrm{T}$. Are antibiotics effective for acute purulent rhinitis? Systematic review and meta-analysis of placebo controlled randomised trials. BMJ 2006;333:279-81. 


\section{What is already known on this topic}

Despite evidence for little or no benefit in many common conditions, antibiotics continue to be overprescribed in primary care, unnecessarily exposing patients to unwanted effects and selecting for resistant organisms

Training in consultation skills can reduce antibiotic prescribing among individually recruited patients over the short term

\section{What this study adds}

It is possible to reduce antibiotic dispensing for practice populations

A flexible blended learning programme led to reductions in practice-wide oral antibiotic dispensing for all causes over the subsequent year

6 Ahovuo-Saloranta A, Rautakorpi U-M, Borisenko OV, Liira H, Williams JW Jr, Makela M. Antibiotics for acute maxillary sinusitis. Cochrane Database Syst Rev 2008;2:CD000243. Smucny J, Fahey T, Becker L, Glazier R. Antibiotics for acute bronchitis. Cochrane Database Syst Rev 2004;4:CD000245.

8 Malhotra-Kumar S, Lammens C, Coenen S, Van Herck K, Goossens H. Effect of azithromycin and clarithromycin therapy on pharyngeal carriage of macrolide-resistant streptococci in healthy volunteers: a randomised, double-blind, placebo-controlled study. Lancet 2007;369:482-90.

9 Cosby JL, Francis N, Butler CC. The role of evidence in the decline of antibiotic use for common respiratory infections in primary care. Lancet Infect Dis 2007;7:749-56.

10 Little P, Gould B, Williamson I, Warner G, Gantley M, Kinmonth A-L. Reattendance and complications in a randomised trial of prescribing strategies for sore throat: the medicalising effect of prescribing antibiotics. BMJ 1997;315:350-2.

11 Butler CC, Hillier S, Roberts Z, Dunstan F, Howard A, Palmer S. Antibiotic-resistan infections in primary care are symptomatic for longer and increase workload: outcomes for patients with E coli UTIs. Br J Gen Pract 2006;56:686-92.

12 Alam MF, Cohen D, Butler C, Dunstan F, Roberts Z, Hillier S, et al. The additional costs of antibiotics and re-consultations for antibiotic-resistant Escherichia coli urinary tract infections managed in general practice. Int $J$ Antimicrob Agents 2009;33:255-7.

13 Ball P, Baquero F, Cars O, File T, Garau J, Klugman K, et al. Antibiotic therapy of community respiratory tract infections: strategies for optimal outcomes and minimized resistance emergence. J Antimicrob Chemother 2002;49:31-40.

14 Butler CC, Dunstan F, Heginbothom M, Mason B, Roberts Z, Hillier S, et al. Containing antibiotic resistance: decreased antibiotic-resistant coliform urinary tract infections with reduction in antibiotic prescribing by general practices. Br J Gen Pract 2007:57:785-92.

15 Seppala H, Klaukka T, Vuopio-Varkila J, Muotiala A, Helenius H, Lager K, et al. The effect of changes in the consumption of macrolide antibiotics on erythromycin resistance in group A streptococci in Finland. Finnish Study Group for Antimicrobial Resistance. N Eng J Med 1997;337:441-6.

16 Guillemot D, Varon E, Bernede C, Weber P, Henriet L, Simon S, et al. Reduction of antibiotic use in the community reduces the rate of colonization with penicillin G-nonsusceptible Streptococcus pneumoniae. Clin Infect Dis 2005;41:930-8.

17 Grijalva CG, Nuorti JP, Griffin MR. Antibiotic prescription rates for acute respiratory tract infections in US ambulatory settings. JAMA 2009;302:758-66.

18 Thompson PL, Spyridis N, Sharland M, Gilbert RE, Saxena S, Long PF, et al. Changes in clinical indications for community antibiotic prescribing for children in the UK from 1996 to 2006: will the new NICE prescribing guidance on upper respiratory tract infections just be ignored? Arch Dis Child 2009;94:337-40.

19 Meropol SB, Chen Z, Metlay JP. Reduced antibiotic prescribing for acute respiratory infections in adults and children. Br J Gen Pract 2009;59:e321-8.

20 Huttner B, Goossens H, Verheij T, Harbarth S. Characteristics and outcomes of public campaigns aimed at improving the use of antibiotics in outpatients in high-income countries. Lancet Infect Dis 2010:10:17-31.

21 Butler CC, Hood K, Verheij T, Little P, Melbye H, Nuttall J, et al. Variation in antibiotic prescribing and its impact on recovery in patients with acute cough in primary care: prospective study in 13 countries. BMJ 2009;338:b2242.

22 Coenen S, Kuyenhoven MM, Butler CC, Van Royen P, Verheij TJ. Variation in European antibiotic use. Lancet 2001;358:1272.

23 Ranij SR, Steinman MA, Shojania KG, Gonzales R. Interventions to reduce unnecessary antibiotic prescribing: a systematic review and quantitative analysis. Med Care 2008;46:847-62.

24 Ostini R, Hegney D, Jackson C, Williamson M, Mackson JM, Gurman K, et al. Systematic review of interventions to improve prescribing. Ann Pharmacother 2009;43:502-13.

25 Martens J, Winkens R, van der Weijden T, de Bruyn D, Severens J. Does a joint development and dissemination of multidisciplinary guidelines improve prescribing behaviour: a pre/post study with concurrent control group and a randomised trial. BMC Health Serv Res 2006;2:145.

26 Van Driel M, Coenen S, Dirven K, Lobbestael J, Janssens I, Van Roven P, et al. What is the role of quality circles in strategies to optimise antibiotic prescribing? A pragmatic cluster-randomised controlled trial in primary care. Qual Saf Health Care 2007;16:197-202.

27 Oxman AD, Thomson MA, Davis DA, Haynes RB. No magic bullets: a systematic review of 102 trials of interventions to improve professional practice. CMAJ 1995;153:1423-31

28 Davis DA, Thompson MA, Oxman AD, Haynes RB. Changing physician performance. A systematic review of the effect of continuing medical education strategies. JAMA 1995;274:700-5.

29 Thomson O'Brien MA, Oxman AD, Davis DA, Haynes RB, Freemantle N, Harvey EL. Educational outreach visits: effects on professional practice and health care outcomes. Cochrane Database Syst Rev 2000;2:CD000409.

30 Finch RG, Metlay JP, Davey PG, Baker LJ. Educational interventions to improve antibiotic use in the community: report from the International Forum on Antibiotic Resistance (IFAR) colloquium, 2002. Lancet Infect Dis 2004;4:44-3.

31 Foy R, MacLennan G, Grimshaw J, Penney G, Campbell M, Grol R. Attributes of clinical recommendations that influence change in practice following audit and feedback. $J$ Clin Epidemiol 2002;55:717-22.

32 Arnold SR, Straus SE. Interventions to improve antibiotic prescribing practices in ambulatory care. Cochrane Database Syst Rev 2005;4:CD003539.
33 Eccles M, Grimshaw J, Walker A, Johnston M, Pitts N. Changing the behavior of healthcare professionals: the use of theory in promoting the uptake of research findings. $J$ Clin Epidemiol 2005;58:107-12.

34 Schiff GD, Galanter WL. Promoting more conservative prescribing. JAMA 2009;301:865-7.

35 Butler CC, Prout H, Kinnersley P, Rollnick S, Edwards A, Elwyn G. Shared decision making and antibiotics in primary care. J Antimicrobial Chemother 2001:48:435-40.

36 Cals JW, Butler CC, Hopstaken RM, Hood K, Dinant GJ. Effect of point of care testing for $\mathrm{C}$ reactive protein and training in communication skills on antibiotic use in lower respiratory tract infections: cluster randomised trial. BMJ 2009;338:b1374.

37 Francis NA, Butler CC, Hood K, Simpson S, Wood F, Nuttall J. Effect of using an interactive booklet about childhood respiratory tract infections in primary care consultations on reconsulting and antibiotic prescribing: a cluster randomised controlled trial. $B M J$ 2009;339:b2885.

38 Butler CC, Rollnick S, Maggs-Rapport F, Pill RM, Stott NCH. Understanding the culture of prescribing: a qualitative study of general practitioners' and patients' perceptions of antibiotics for sore throats. BMJ 1998;317:637-42.

39 Wood F, Simpson S, Butler CC. Socially responsible antibiotic choices in primary care: a qualitative study of GPs' decisions to prescribe broad-spectrum and fluroquinolone antibiotics. Fam Pract 2007;24:427-34.

40 Grol R, Baker R, Moss F. Quality improvement research: understanding the science of change in health care. Qual Saf Health Care 2002;11:110-1.

41 Butler CC, Rollnick S, Kinnersley P, Jones A, Stott NCH. Reducing antibiotics for respiratory tract symptoms in primary care; consolidating "why" and considering "how." Br J Gen Pract 1998:48:1865-70.

42 Rollnick S, Mason P, Butler CC. Health behaviour change: a guide for practitioners Churchill Livingstone, 1999

43 Simpson SA, Butler CC, Hood K, Cohen D, Dunstan F, Evans MR, et al. Stemming the tide of antibiotic resistance (STAR): a protocol for a trial of a complex intervention addressing the "why" and "how" of appropriate antibiotic prescribing in general practice. BMC Fam Pract 2009;10:20.

44 Carter B, Hood K. Balance algorithm for cluster randomized trials. BMC Med Res Methodol 2008;8:65.

45 Xiao L, Lavori PW, Wilson SR, Ma J. Comparison of dynamic block randomization and minimization in randomized trials: a simulation study. Clin Trials 2011;8:59-69.

46 Rollnick S, Miller WR, Butler C. Motivational interviewing in health care. Guildford Press, 2008.

47 Cals JW, Scheppers NA, Hopstaken RM, Hood K, Dinant GJ, Goettsch, et al. Evidence based management of acute bronchitis; sustained competence of enhanced communication skills acquisition in general practice. Patient Educ Couns 2007;68:270-8.

48 Rollnick S, Seale C, Kinnersley P, Rees M, Butler C, Hood K. Developing a new line of patter: can doctors change their consultations for sore throat? Med Educ 2002;36:678-81.

49 Rollnick S, Kinnersley P, Butler C. Context-bound communication skills training: development of a new method. Med Educ 2002;36:377-83.

50 Lambert MF, Masters GA, Brent SL. Can mass media campaigns change antimicrobial prescribing? A regional evaluation study. J Antimicrobial Chemother 2007;59:537-43.

51 Curtis L. Unit costs of health and social care. Personal Social Services Research Unit, University of Kent, Canterbury, 2008.

52 British Medical Association and the Royal Pharmaceutical Society of Great Britain. British National Formulary. 54th ed. BMA and the Royal Pharmaceutical Society of Great Britain, 2007.

53 Mclsaac W, Goel V, To T, Low DE. The validity of a sore throat score in family practice. CMAJ 2000;163:811-5.

54 Eldridge S, Kerry S, Torgerson DJ. Bias in identifying and recruiting participants in cluster randomised trials: what can be done? BMJ 2009;339-b4006.

55 Welschen I, Kuyvenhoven M, Hoes A, Verheij TJ. Effectiveness of a multiple intervention to reduce antibiotic prescribing for respiratory tract symptoms in primary care: randomised controlled trial. BMJ 2004;329:431.

56 Bekkers MJ, Simpson SA, Dunstan F, Hood K, Hare M, Evans CC, et al. Enhancing the quality of antibiotic prescribing in primary care: qualitative evaluation of a blended learning intervention. BMC Fam Pract 2010;11:34.

57 McNulty CA, Nichols T, Boyle PJ, Woodhead M, Davey P. The English antibiotic awareness campaigns: did they change the public's knowledge of and attitudes to antibiotic use? $J$ Antimicrobial Chemother 2010;65:1526-33.

\section{Accepted: 05 November 2011}

\section{Cite this as: BMJ 2012;344:d8173}

This is an open-access article distributed under the terms of the Creative Commons Attribution Non-commercial License, which permits use, distribution, and reproduction in any medium, provided the original work is properly cited, the use is non commercial and is otherwise in compliance with the license. See: $\mathrm{http}: / /$ creativecommons.org/licenses/by$\mathrm{nc} / 2.0 /$ and http://creativecommons.org/licenses/by-nc/2.0/legalcode. 


\section{Tables}

Table 1| Summary of demographic features of practices undergoing educational programme aimed at reducing antibiotic prescribing in primary care. Figures are numbers (percentages) unless stated otherwise

\begin{tabular}{lcc} 
& Intervention & Control \\
Practices $^{*}$ & 34 & 34 \\
\hline Clinicians in practices $^{*}$ & 159 & 155 \\
\hline Consenting clinicians $^{*}$ & 139 & 124 \\
\hline Mean list size (at baseline) & 7050 \\
\hline Participating clinicians $\dagger$ & 127 & 115 \\
\hline Male clinicianst & $77(61)$ & $76(66)$ \\
\hline Age (years)†: & $7(6)$ & $1(1)$ \\
\hline $25-30$ & $35(28)$ & $24(21)$ \\
\hline $31-40$ & $48(38)$ & $56(49)$ \\
\hline $41-50$ & $35(28)$ & $31(27)$ \\
\hline $51-60$ & $2(2)$ & $3(3)$
\end{tabular}

${ }^{*}$ Based on data for all randomised practices.

†Based on data for participating clinicians (either completing online training for intervention group or clinicians in control group for whom data were available). 
Table 2| Summary results of antibiotic dispensing (primary outcome), dispensing cost per practice, and rate of admission to hospital in practices undergoing educational programme aimed at reducing antibiotic prescribing in primary care

\begin{tabular}{|c|c|c|c|c|c|c|}
\hline \multirow[b]{2}{*}{ Outcome } & \multicolumn{2}{|c|}{ Mean at baseline } & \multicolumn{2}{|c|}{ Mean at follow-up } & \multirow{2}{*}{$\begin{array}{l}\text { \% reduction in intervention } \\
\text { group relative to control } \\
\text { group }(95 \% \mathrm{Cl})\end{array}$} & \multirow[b]{2}{*}{$P$ value } \\
\hline & Control & Intervention & Control & Intervention & & \\
\hline All antimicrobials $†$ & 669.0 & 678.1 & 681.1 & 664.0 & $4.2(0.6$ to 7.7$)$ & 0.02 \\
\hline \multicolumn{7}{|l|}{ Broad spectrum penicillinst: } \\
\hline All $†$ & 254.3 & 252.6 & 249.6 & 238.9 & $4.7(-1.6$ to 10.7$)$ & 0.14 \\
\hline Amoxicillin† & 215.5 & 215.8 & 211.5 & 203.9 & $4.7(-1.5$ to 10.6$)$ & 0.13 \\
\hline Co-amoxiclav $\dagger$ & 36.0 & 34.6 & 36.3 & 33.7 & $7.3(-5.1$ to 7.3$)$ & 0.23 \\
\hline Phenoxymethylpenicillin† & 45.8 & 53.3 & 47.3 & 49.5 & $7.3(0.4$ to 13.7$)$ & 0.04 \\
\hline Cephalosporins $†$ & 53.7 & 50.0 & 55.6 & 49.5 & $2.3(-8.0$ to 11.6$)$ & 0.65 \\
\hline Macrolides† & 73.9 & 76.4 & 76.7 & 73.7 & $7.7(1.1$ to 13.8$)$ & 0.02 \\
\hline Quinolones† & 22.0 & 20.9 & 23.7 & 20.8 & $8.3(-2.9$ to 18.5$)$ & 0.14 \\
\hline $\begin{array}{l}\text { Penicillinase-resistant } \\
\text { penicillins } †\end{array}$ & 67.8 & 76.3 & 67.5 & 76.2 & $-3.4(-12.3$ to 4.8$)$ & 0.43 \\
\hline Trimethoprim† & 65.5 & 63.2 & 70.6 & 66.6 & $4.3(-2.4$ to 8.9$)$ & 0.24 \\
\hline Tetracyclines† & 57.0 & 57.3 & 60.3 & 58.5 & $4.7(-1.5$ to 10.6$)$ & 0.22 \\
\hline Hospital admissions $¥$ & 8.7 & 7.7 & 8.0 & 7.5 & $-1.9(-13.2$ to 8.2$)$ & 0.72 \\
\hline Dispensing cost/practice $(£)$ & 2254.6 & 2199.7 & 2252.3 & 2078.9 & $5.5(-0.4$ to 11.4$)$ & 0.07 \\
\hline
\end{tabular}

*Difference between means in intervention group and control group as percentage of mean in control group.

†Annual No of dispensed units for oral antibiotics per 1000 registered patients.

$\ddagger$ Annual No of hospital episodes for possible respiratory tract infections and complications of common infections per 1000 registered patients. 
Table 3| Re-consultation rates for respiratory tract infections within seven, 14, and 31 days in practices undergoing educational programme aimed at reducing antibiotic prescribing in primary care

\begin{tabular}{|c|c|c|c|c|}
\hline \multirow[b]{2}{*}{ Re-consultation rate ${ }^{*}$} & \multicolumn{2}{|c|}{ Median (IQR) } & \multirow[b]{2}{*}{ Median difference (95\% Clł) } & \multirow[b]{2}{*}{$\mathbf{P}$ value§ } \\
\hline & Intervention $(n=20) \dagger$ & Control $(n=17) \dagger$ & & \\
\hline Within 7 days & $2.66(1.88-4.25)$ & $3.35(2.16-4.31)$ & $-0.65(-1.69$ to 0.55$)$ & 0.446 \\
\hline Within 14 days & $5.10(4.70-7.92)$ & $6.43(4.04-7.84)$ & $-1.33(-2.12$ to 0.74$)$ & 0.411 \\
\hline Within 31 days & 9.06 (7.53-12.62) & 11.38 (7.39-14.05) & $-2.32(-4.76$ to 1.95$)$ & 0.503 \\
\hline
\end{tabular}

IQR=interquartile range.

*Median No of individuals who reconsulted for respiratory tract infection per 1000 registered patients.

†Values in each group refer to subset of intervention practices for which data on reconsultation were available.

$\ddagger$ Computed with bootstrapping methods.

§From Mann-Whitney U test. 
Table 4 | Costs of STAR educational programme aimed at reducing antibiotic prescribing in primary care

\begin{tabular}{|c|c|c|c|}
\hline Activity & Units & Unit cost $(£)$ & Cost $(£)$ \\
\hline \multicolumn{4}{|l|}{ Administration } \\
\hline Administration & 350.6 hours & Various* & 4320 \\
\hline Materials and postage & Various & Varioust & 434 \\
\hline Total & - & - & 4754 \\
\hline \multicolumn{4}{|l|}{ Seminar preparation } \\
\hline Project staff preparation time & 45.5 hours & Various* & 1598 \\
\hline Training trainers & 14 hours & Various* & 364 \\
\hline Preparing practice level data (Health Solution Wales staff) & 5 days & $115^{\star}$ & 574 \\
\hline Totalł & - & - & 2536 \\
\hline \multicolumn{4}{|l|}{ Seminar delivery } \\
\hline Trainers' fee & 33 seminars & 500 & 16500 \\
\hline Trainers' travel/expenses & Claim forms & - & 1010 \\
\hline Totalf & - & - & 17510 \\
\hline \multicolumn{4}{|l|}{ Cost to practices } \\
\hline Physician time (seminar) & $126 @ 1.5$ hours & $104 \S$ & 19656 \\
\hline Nurse practitioner time (seminar) & $2 @ 1.5$ hours & $28 \rrbracket$ & 84 \\
\hline Physician time (logged on) & 496.40 hours & $104 \S$ & 51626 \\
\hline Nurse practitioner time (logged on) & 10.51 hours & $28 \rrbracket$ & 294 \\
\hline Total cost of trainee time $\ddagger$ & - & - & 71659 \\
\hline Total cost of STAR training (33 practices) $\ddagger$ & - & - & 96460 \\
\hline Mean (SD) cost per practice & - & - & $2923(1187)$ \\
\hline
\end{tabular}

*Staff salaries uplifted by $22.5 \%$ to include on costs (national insurance, etc). †University admin dept/Royal mail.

$\ddagger$ Totals rounded.

$\S$ Per hour GMS activity, with qualifications excluding direct care staff costs. ${ }^{51}$

IPer hour with qualifications (no client contact). ${ }^{51}$ 


\section{Figures}

\begin{tabular}{|c|c|c|c|c|c|}
\hline$\left\{\begin{array}{l}\text { STAR } \\
\text { Educational Programme }\end{array}\right.$ & Programme & About Us & Resources & Portfolio & Help \\
\hline Programme & \multicolumn{5}{|c|}{ You are logged in as: Admin Admin Logout? } \\
\hline Part & \multicolumn{3}{|c|}{ Module } & \multicolumn{2}{|r|}{ Status } \\
\hline $\begin{array}{l}\text { PART 1: e-learning } \\
\text { The challenge }\end{array}$ & \multicolumn{3}{|c|}{ Introduction } & & \$) Completed \\
\hline & \multicolumn{3}{|c|}{ What would you do? } & & \) Completed (O) \\
\hline & \multicolumn{3}{|c|}{ What do you think? } & & \) Completed O \\
\hline & \multicolumn{3}{|c|}{ Some perspectives } & & \) Completed (0) \\
\hline & \multicolumn{3}{|c|}{ Evidence } & & 》) Completed (O) \\
\hline & \multicolumn{3}{|c|}{ Summary } & & D) Completed () \\
\hline $\begin{array}{l}\text { PART 2: } \\
\text { Portfolio Task } 1\end{array}$ & \multicolumn{3}{|c|}{ Upload 4 consultations } & & \Completed \\
\hline $\begin{array}{l}\text { PART 3: } \\
\text { Brief Seminar }\end{array}$ & \multicolumn{3}{|c|}{ Seminar } & & 》Completed \\
\hline \multirow{4}{*}{$\begin{array}{l}\text { PART 4: e-learning } \\
\text { Inside the Consultation }\end{array}$} & \multicolumn{3}{|c|}{ What would you do? } & & \$) Completed () \\
\hline & \multicolumn{3}{|c|}{ Core tasks } & & D) Completed \\
\hline & \multicolumn{3}{|c|}{ What do you think? } & & \$) Completed \\
\hline & \multicolumn{3}{|c|}{ Wrap up } & & D) Completed (O) \\
\hline $\begin{array}{l}\text { PART 5: } \\
\text { Portfolio Task } 2\end{array}$ & \multicolumn{3}{|c|}{ Three of the Best } & & D) Completed \\
\hline $\begin{array}{l}\text { PART 6: } \\
\text { Using the STAR network }\end{array}$ & \multicolumn{3}{|c|}{ Web forum } & & \) Completed \\
\hline $\begin{array}{l}\text { PART 7: e-learning } \\
\text { Booster session }\end{array}$ & \multicolumn{3}{|c|}{ Booster session } & & D) Completed \\
\hline
\end{tabular}

Fig 1 Screenshot of the whole programme

\section{STAR}

Programme

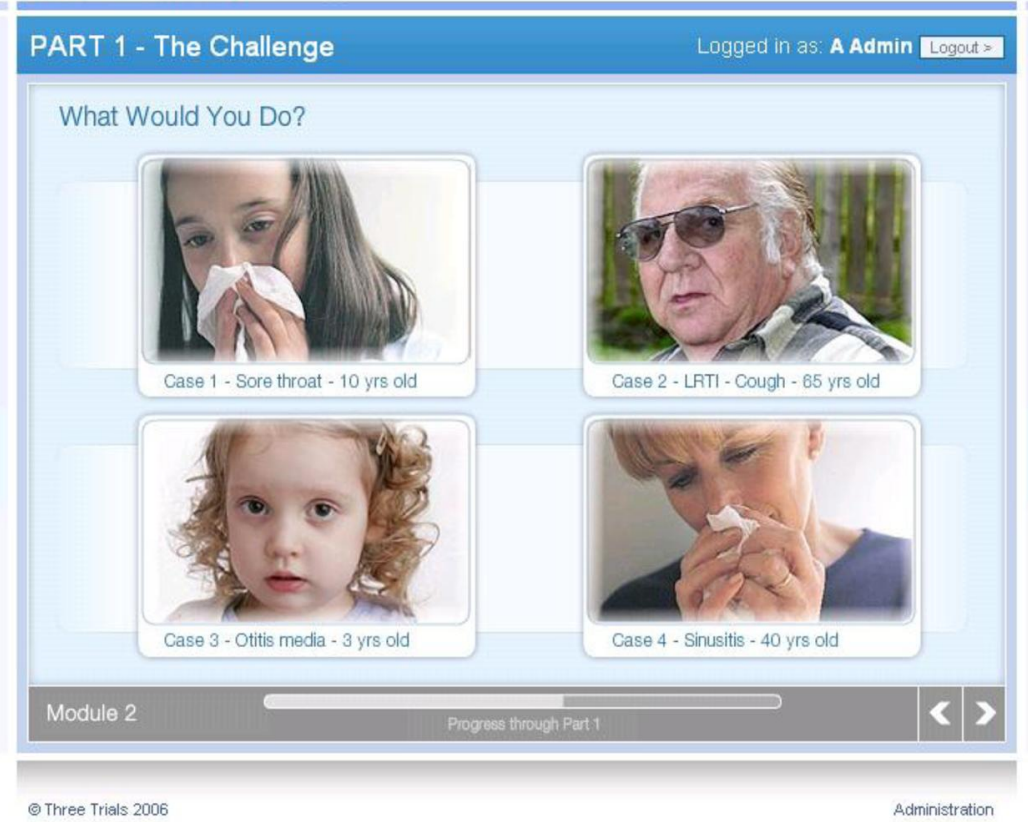

Fig 2 Screenshot of the "What would you do?" section 


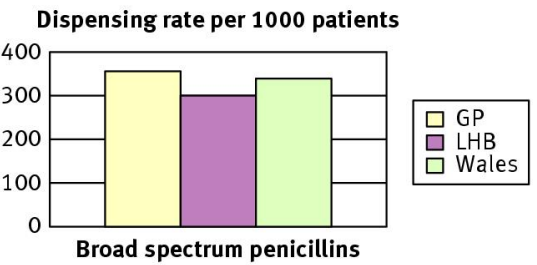

$\%$ urine samples resistant

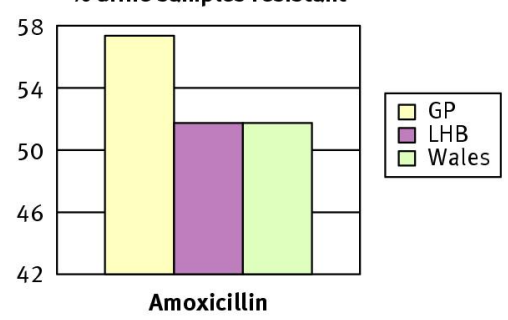

$\%$ urine samples resistant

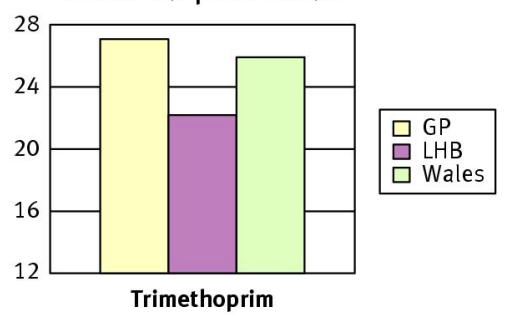

Fig 3 Example of data fed back during practice based seminars: "Your practices' antibiotic prescribing and resistance in urine samples compared to your Local Health Board (LHB) and all Wales, averaged over seven years"

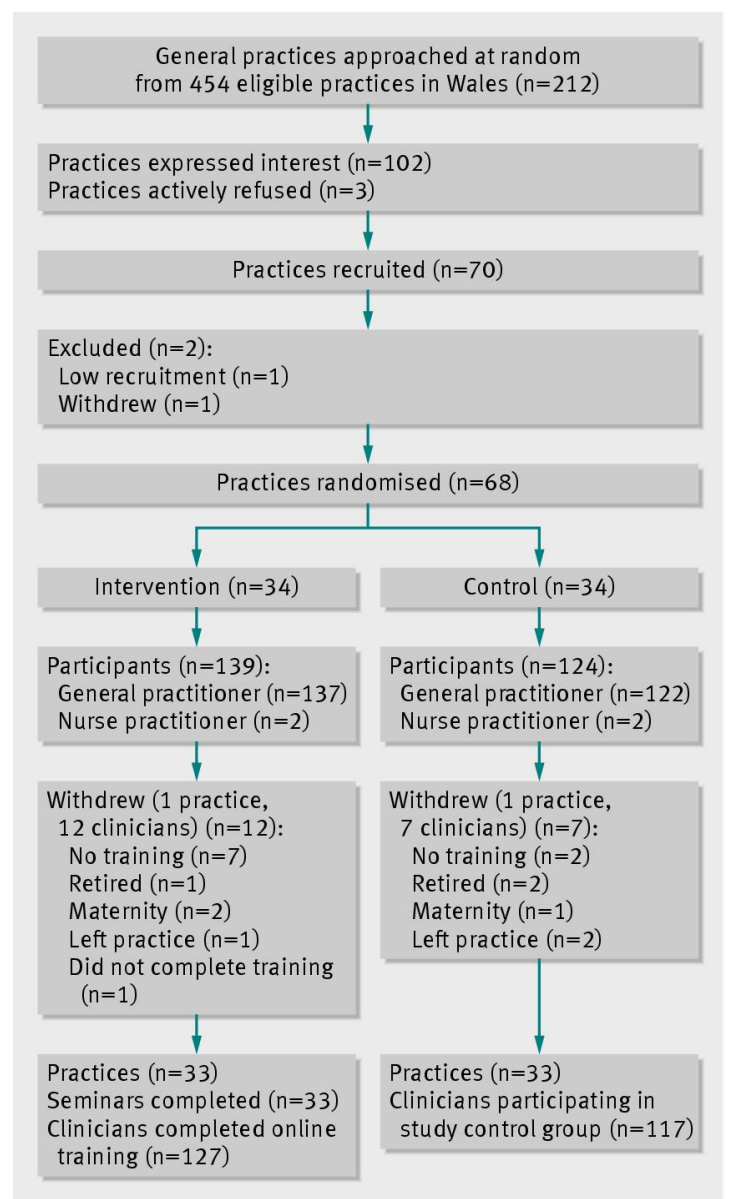

Fig 4 Participant flow diagram 